\title{
Philosophiques
}

\section{La définition de la simultanéité pour des corps en mouvements rectilignes et pour ceux en mouvements circulaires}

\section{Jacques Bouet}

Volume 24, numéro 2, automne 1997

URI : https://id.erudit.org/iderudit/027456ar

DOI : https://doi.org/10.7202/027456ar

Aller au sommaire du numéro

Éditeur(s)

Société de philosophie du Québec

ISSN

0316-2923 (imprimé)

1492-1391 (numérique)

Découvrir la revue

Citer cet article

Bouet, J. (1997). La définition de la simultanéité pour des corps en mouvements rectilignes et pour ceux en mouvements circulaires. Philosophiques, 24(2),

341-344. https://doi.org/10.7202/027456ar
Résumé de l'article

Une théorie physique s'appuie généralement sur un modèle physique. Un modèle habituel est formé par des corps en mouvements rectilignes uniformes. Il est tout aussi légitime de considérer un autre modèle formé par des corps en mouvements circulaires uniformes. Les conclusions concernant la simultanéité, l'écoulement du temps, obtenues à partir du premier modèle, ne semblent pas pouvoir être étendues au second. 


\title{
LA DÉFINITION DE LA SIMULTANÉIIÉ POUR DES CORPS EN MOUVEMENTS RECTIIIGNES ET POUR CEUX EN MOUVEMENIS CIRCULAIRES
}

\author{
PAR \\ JACOUES BOUET ${ }^{*}$
}

\begin{abstract}
RÉSUMÉ: Une théorie physique s'appuie généralement sur un modèle physique. Un modèle habituel est formé par des corps en mouvements rectilignes uniformes. Il est tout aussi légitime de considérer un autre modèle formé par des corps en mouvements circulaires uniformes. Les conclusions concernant la simultanéité, lécoulement du temps, obtenues à partir du premier modèle, ne semblent pas pouvoir être étendues au second.

ABSTRACT: A physical theory is generally based on a physical model. One ordinary model consists of bodies in uniform rectilinear motion. It is just as legitimate to consider another model consisting of bodies in uniform circular motion. It seems that the conclusions on simultaneity obtained from the uniform rectilinear model cannot be extended to the uniform circular model.
\end{abstract}

\section{Le problème de la simultanéité pour des corps en mouvements rectilignes uniformes}

A. Einstein ${ }^{1}$ a mis en doute la possibilité d'établir empiriquement la simultanéité d 'évé nements qui se produisent sur des mobiles en mouvements relatifs. Pour établir sa démonstration, il rassemble et met en relation un certain nombre d'éléments :

un ensemble de constatations expérimentales relatives à la propagation de la lumière. La vitesse de celle-ci paraît indépendante de la vitesse de la source é mettrice et de celle du récepteur ;

- un modèle physique concernant la matière. Ce modèle cst constitué par des corps en mouvements rectilignes uniformes. Ces corps transportent des horloges et des observateurs ;

- une méthodologie opérationnaliste. Les observateurs considérés ne peuvent communiquer entre eux qu'en échangeant des signaux lumineux. Pour chaque observateur, les mesures qu'il peut obtenir de la marche des

NDLR L'intervention de M. Bouet a été adressée à Philosophiques en guise de commentaire au débat ouvert sur la physique contemporaine et le renouvellement de la métaphysique. Débat reproduit dans le $\mathrm{n}^{\circ} 2$ du vol. XXII sous la rubrique Table ronde et intitulé “ La philosophie des sciences au Québec: trois contributions majeures n.

1. A. Einstein, Sur l'électrodynamique des corps en mouvements, trad. par M. Solovine, Paris, Gauthier-Villars, 1955. 
autres horloges sont donc complètement sous la dépendance des propriétés remarquables de la propagation de la lumière.

A. Einstein établit alors que si l'on respecte toutes les conditions posées, il existe un temps propre pour chaque système inertial sans qu'on puisse en privilégier un.

\section{L'extension de la notion de temps propre aux modéles en mouvements circulaires uniformes}

Ce problème a été examiné par E. Borel² :

Pour définir l'unité de temps, nous pouvons choisir une horloge céleste é galement visible de Mars et de la Terre, par exemple, la durée de la ré volution de l'un des satellites de Jupiter $[\ldots]$ La question qui se pose maintenant est de savoir si la représentation du système solaire que se formera l'observate ur martien sera exactement la même que celle que se formera l'observateur terrestre $[\ldots]$ or, il est clair que le rayon lumineux qui nous parvient du satellite de Jupiter a mis un certain temps pour parvenir de cet astre [...] Toute notre connaissance de l'espace planétaire suppose clon $c$, outre les hypothèses déjà mises en évidence, cette hypoth èse essentielle, que la vitesse de la lumière dans le vide nous est connue, c'est-à- clire est constante [...] Or, le fait que les astres observés sont en mouvement les uns par rapport aux autres introduit alors une difficult é essentielle qui est le nœud même des théories nouvelles de la relativité $[\ldots]$

La théorie de la relativité restreinte ne s'applique d'ailleurs en toute rigueur que pour des groupes d 'observateurs dont le mouvement relatif est un mouvement rectiligne et uniforme; mais tout mouvement relatif de deux portions assez petites de l'espace peut ètre regarclé, s'il est continu et envisagé pendant un temps sulfisamment court comme rectiligne et uniforme.

II y a dans ce contexte l'ébauche du raisomnement qui conduit à étendre la notion de temps propre du mouvement rectiligne au mouvement circulaire considéré pendant une courte durée, puis de cette fraction de cercle à un cercle entier, puis finalement au mouvement circulaire uniforme.

Cette extension est-elle indiscutable? Ne passe-t-on pas d'un mod èle où certaines conclusions s'imposent, à un autre modèle où elles ne s'imposent peut-être pas?

\section{Le problème de la simultanéité pour des corps en mouvements circulaires uniformes}

Considérons le modèle astronomique proposé par E. Borel, soit done un modèle constitué par des corps en mouvements circulaires uniformes autour d'un point comniun et ceci, pendant un très grand nombre de révolutions. Au bout d'une révolution ou de quelques milliers de révolutions, l'indétermination entre les mesures martiennes et terrestres sur la position absolue du satellite de Jupiter sera toujours la même. Lorsque ce dernier aura effectuée quelques millions de révolutions, les observateurs martiens et terrestres seront d'accord sur ce nombre avec une indétermination ne portant que sur une fraction infime de la durée de révolution du satellite.

La précision sur la simultanéité d'un événement (datation de l'éclipse d'un satellite par exemple) pour deux observateurs en mouvements relatifs.

2. E. Borel, L'Espace et le Temps, Paris, PUF, 1949, p. 65-67, 135. 
peut donc devenir, en valeur relative, infiniment grande. Par contre, les problèmes que pose, pour la détermination spatiotemporelle de cet événe. ment, la composition de la vitesse de la lumière avec celle des corps émetteurs ou récepteurs, deviennent, eux, de plus en plus secondaires au cours du fonctionnement du modèle astronomique retenu. On peut modifier légèrement ce modèle, doter chaque corps en mouvements circulaires de sa propre horloge (la Terre et Mars ont alors chacune un satellite). Les résultats précédents ne sont pas modifiés. Les observateurs situés sur ces deux planètes finiront toujours par totaliser deux nombres identiques pour les révolutions de ces deux satellites.

Accepter l'extension du concept de temps propre, d'un modèle où les horloges sont en mouvements rectilignes, à un modèle où elles sont en mouvements circulaires, comme le suggère E. Borel, c'est accepter en conséquence le paradoxe suivant : des observateurs situés sur Terre et sur Mars doivent finir par constater, en échangeant entre eux des informations cumulées, qu'ils ont vu des nombres différents de révolutions pour, par exemple, un satellite de la Terre.

Le monde ne nous est donné qu'une fois avec l'inertie et la gravitation.

Pour démontrer pour la première fois l'existence de l'inertie et ses propriétés remarquables, Galilée utilise un modèle célèbre qui mélange d'une manière empirique et opérationnaliste inertie et gravitation. II considère une bille lancée sur la surface d'un globe terrestre parfaitement sphérique et dépourvu de forces de frottement. La vitesse acquise par cette bille se maintiendra éternellement, mais son mouvement sera circulaire. En effet, motto retto impossible per natura (voir à ce sujet A. Koyré ${ }^{3}$.

Le choix délibéré d'observateurs, d'horloges en mouvements circulaires uniformes autour d'un point commun est tout aussi admissible que le choix d'observateurs, d'horloges en mouvements rectilignes uniformes. L'option choisie permet en privilégiant le rôle des mouvements circulaires de ne faire jo uer à la propagation de la lumière et aux problèmes qu'elle pose qu 'un rôle accessoire, on n'introduit ainsi dans le modèle physique considéré qu'une indétermination constante et non cumulative au cours du processus de mesure envisagé. La non-existence d'un ether universel, de signaux se déplaçant plus vite que la lumière, la constance de la vitesse de celle-ci pour tous les observateurs n'ont pas les mêmes conséquences pour la reconstruction de l'écoulement du temps pour des observateurs selon qu'ils sont situés sur des corps en mouvements rectilignes ou circulaires.

3. A. Koyré, Études galiléennes, Paris, Hermann, 1966, p. 238. 
Dans un article récent, Mc Call ${ }^{4}$ pose la question de savoir s'il est possible de donner une description précise et scientifique de l'écoulement du temps. Le modèle que nous présentons est certes ancien, mais moyenn ant quelques précisions, il permet de répondre à cette question à l'échelle macroscopique. Les mouvements de rotation et de révolution du globe terrestre s'effectuent par rapport aux étoiles, à l'ensemble des autres corps de l'univers. Le décompte de ces mouvements permet d'identifier une caractéristique réelle du monde qui correspond à la notion de passage temporel.

Mennecy, France

4. S. Mc Call, a Réponse à Yvon Gauthier, Louis Marchand et Serge Robert ", Philosophiques, vol. XXII, n², 1995, p. 500. 